\title{
Recovery of Mature Hepatocytic Phenotype following Bile Ductular Transdifferentiation of Rat Hepatocytes
} in Vitro

\author{
Masayuki Sone, ${ }^{*}$ Yuji Nishikawa, ${ }^{\dagger}$ \\ Yasuharu Nagahama, ${ }^{\dagger}$ Eriko Kumagai, ${ }^{*}$ \\ Yuko Doi, ${ }^{\ddagger}$ Yasufumi Omori, ${ }^{*}$ Toshiaki Yoshioka, ${ }^{*}$ \\ Takuo Tokairin, ${ }^{*}$ Masayuki Yoshida, ${ }^{*}$ \\ Toshihiro Sugiyama, ${ }^{\S}$ and Katsuhiko Enomoto* \\ From the Departments of Molecular Pathology and Tumor \\ Pathology* and Biochemistry ${ }^{\S}$ and the Central Research \\ Laboratory, ${ }^{\ddagger}$ Akita University Graduate School of Medicine, Akita; \\ and the Division of Tumor Pathology, ${ }^{\dagger}$ Department of Pathology, \\ Asabikawa Medical University, Asabikawa, Japan
}

We previously demonstrated that mature rat hepatocytes transdifferentiate to bile ductular cells when cultured in a three-dimensional collagen-rich matrix. Here, we show that the phenotype of transdifferentiated hepatocytes can be reversed by modulating culture conditions. Spheroidal aggregates of hepatocytes were cultured within a collagen gel matrix in the presence of serum and tumor necrosis factor- $\alpha$. Spheroids transformed into ductular structures composed of small cuboidal cells, lost the expression of hepatocytic markers, whereas aberrantly expressed bile ductular markers. The transdifferentiated cells were then retrieved from the gels, plated on surfaces coated with a basement membrane-like material, and cultured in serum-free media. Cells spontaneously formed spheroidal aggregates and recovered hepatocytic phenotype. Dexamethasone (Dex), which suppressed the phosphorylation of ERK and Jun N-terminal kinase, facilitated the recovery, and the combination with interleukin- 6 or oncostatin $M$ resulted in the recovery of hepatocyte nuclear factor $4 \alpha$ protein expression and the typical hepatocytic morphology, and a decrease in the expression of bile ductular markers. A cDNA microarray analysis revealed that the hepatocyte-specific mRNA expression profile was recovered in these cells. Our results demonstrate that hepatocytes are able to recover their phenotypes following bile ductular transdifferentiation, suggesting that hepatocytic and bile ductular phenotypes may be mutually reversible. (AmJ Pathol 2012, 181:2094-2104; http://dx.doi. org/10.1016/j.ajpath.2012.08.034)

The appearance of irregular bile ductular structures is frequently observed in various chronic liver diseases associated with fibrosis. ${ }^{1,2}$ This process is called the atypical ductular reaction, and it has been interpreted as a regenerative proliferation of so-called hepatic progenitor cells, which are believed to be present in the vicinity of the portal tract. ${ }^{3,4}$ However, this reaction might also be due to the ductular metaplasia of hepatocytes. ${ }^{1}$ Supporting this hypothesis, the ductular reaction can be induced in the centrilobular area in rodent chronic liver injury models induced by $\mathrm{CCl}_{4}$ or thioacetamide, where no bile ducts or hepatic progenitors exist (Nishikawa et al, unpublished observation) (see Supplemental Figure S1 at http://ajp.amjpathol.org). Furthermore, in chronic congestion of the human liver, the appearance of ductular hepatocytes with bile duct-specific cytokeratin has been observed in the centrilobular area. ${ }^{5}$ This type of ductular reaction has been recently described and interpreted as a result of dedifferentiation of mature hepatocytes due to hypoxia. ${ }^{6}$ It is important to precisely understand the origin and pathogenesis of the atypical ductular reaction because the prevention of its progression should be beneficial to maintain the proper functioning of the liver if the reaction is due to ductular metaplasia of hepatocytes.

We previously reported that mature hepatocytes could transdifferentiate into bile ductular cells when embedded within a type I collagen gel matrix and cultured in the

Supported by the Ministry of Education, Culture, Sports, Science and Technology of Japan (No. 13670204, 16590303, and 18590362 to Y.N.). Accepted for publication August 20, 2012.

Supplemental material for this article can be found on http://ajp. amjpathol.org or at $h t t p: / / d x . d o i . o r g / 10.1016 / j$. ajpath.2012.08.034.

Address reprint requests to Yuji Nishikawa, M.D., Ph.D., Division of Tumor Pathology, Department of Pathology, Asahikawa Medical University, Higashi 2-1-1-1 Midorigaoka, Asahikawa, Hokkaido 078-8510, Japan. E-mail: nishikwa@asahikawa-med.ac.jp. 
presence of epidermal growth factor and insulin. ${ }^{7,8}$ This phenotypic change is characterized by a loss of hepatocytic differentiation markers and the expression of bile ductular markers, but it is not associated with the reexpression of markers of hepatic progenitor cells, such as delta-like. Other investigators have also reported similar findings regarding the ductular transdifferentiation of hepatocytes both in vitro and in vivo. ${ }^{9-11}$ Recently, we found that tumor necrosis factor- $\alpha$ (TNF- $\alpha$ ) significantly enhanced ductular transdifferentiation (Nishikawa et al, unpublished data).

An important question to be addressed is the reversibility of the bile ductular transdifferentiation of hepatocytes. In our observation of $\mathrm{CCl}_{4}$-induced liver fibrosis, the centrilobular ductular reaction could diminish after cessation of injurious stimuli (see Supplemental Figure S1 at http://ajp.amjpathol.$o r g$ ). If the process is reversible, it may be possible to recover the hepatocytic phenotype in some types of ductular reactions, providing a rationale for the development of a new therapeutic approach targeted to ductular reaction in chronic liver diseases. In preliminary experiments, we retrieved the transdifferentiated hepatocytes from a collagen gel matrix and plated them on Matrigel, a basement membrane matrix that is known to be the most suitable for the maintenance of hepatocytic differentiation in vitro. ${ }^{12}$ When cultured in serum-free medium, the hepatocytes gradually recovered their expression of albumin and tyrosine aminotransferase (TAT) mRNA. Although our data indicated that the transdifferentiation process may be reversible, the degree of re-differentiation was low, and the cells remained small and morphologically similar to ductular cells rather than to mature hepatocytes.

In this study, we attempted to find the condition under which transdifferentiated hepatocytes recovered mature phenotypes. Our previous experiments showed that IL-6 inhibited the loss of albumin expression in hepatocytes in the collagen gel matrix. Furthermore, the combination of dexamethasone (Dex) and oncostatin M (OSM), an IL-6 family cytokine, has been shown to be important for the maturation of mice hepatoblasts in vitro. ${ }^{13,14}$ Thus, we studied the effect of Dex and various cytokines on the recovery of hepatocytic phenotypes in our in vitro model, and found that both OSM and IL-6 could cause the mature hepatocytic phenotypes, if used in combination with Dex. Our results indicate that the ductular transdifferentiation of hepatocytes is reversible and provides insights into the mutual phenotypic plasticity between hepatocytes and bile ductular cells.

\section{Materials and Methods}

\section{Chronic Liver Injury Induced by $\mathrm{CCl}_{4}$}

Chronic liver injury induced by $\mathrm{CCl}_{4}$ is characterized by centrilobular fibrosis with ductular reaction. To examine the reversibility of ductular reaction, a 1:1 mixture of $\mathrm{CCl}_{4}$ and olive oil was administered to male F344 rats (7 weeks old) by gavage at a dose of $2 \mathrm{~mL} / \mathrm{kg}$ twice per week for 8 weeks, followed by 3 weeks of recovery. The animals were sacri- ficed, and the liver tissues were fixed in phosphate-buffered $10 \%$ formalin, embedded in paraffin, and sectioned.

\section{Isolation of Rat Hepatocytes}

Hepatocytes were isolated from male Albumin-DsRed2 transgenic rat livers (10 to 20 weeks old) by the collagenase perfusion method. In situ two-step collagenase liver perfusion and cell isolation were performed as previously described. ${ }^{7,8}$ Albumin-DsRed2 rats, which express DsRed2 fluorescence under the control of the albumin enhancer/promoter, were used to monitor the level of albumin expression in hepatocytes. ${ }^{15}$ The protocols for animal experimentation were approved by the Animal Research Committee, Akita University. All animal experiments adhered to the criteria outlined in the Guide for the Care and Use of Laboratory Animals prepared by the National Academy of Sciences and published by the NIH (NIH Publication No. 86-23, revised 1996).

\section{Flow Cytometric Analysis}

To examine whether the isolated hepatocyte fraction contained liver stem cell or progenitor cells, we performed flow cytometric analysis for several markers of liver stem cells. Isolated hepatocytes were fixed with phosphate-buffered $2 \%$ paraformaldehyde and then with 90\% methanol. Fetal liver cells, separated from E17.5 livers by gentle mixing in collagenase solution, were used as positive controls. Cells were incubated with primary antibodies (1:400 dilution in PBS with 2\% fetal bovine serum) for 1 hour at $4^{\circ} \mathrm{C}$. The cells were washed three times in fetal bovine serum-PBS and incubated with fluorescein isothiocyanate-conjugated antirabbit IgG antibody (1:400 dilution) for 30 minutes. The cells were washed and immediately analyzed with a FACScan (Becton Dickinson, Franklin Lakes, NJ). The primary antibodies used were anti- $\alpha$-fetoprotein (Proteintech, Chicago, IL), anti-DLK1 (Proteintech), anti-EpCAM (Proteintech), and anti-Thy1 (Novus Biologicals, Littleton, CO).

\section{Three-Dimensional Collagen Gel Culture of Rat Hepatocytes}

Isolated hepatocytes were plated onto positively charged plastic dishes (Primaria, Becton-Dickinson) to form spheroidal aggregates. Cells were cultured in serum-free Williams' Medium E supplemented with $10 \mathrm{mmol} / \mathrm{L}$ nicotinamide, $10 \mathrm{ng} / \mathrm{mL}$ epidermal growth factor (Roche Diagnostics, Mannheim, Germany), $10^{-7} \mathrm{~mol} / \mathrm{L}$ insulin (Sigma-Aldrich, St. Louis, MO), $4.2 \mathrm{mmol} / \mathrm{L} \mathrm{NaHCO}_{3}$, and penicillin/streptomycin $(100 \mathrm{IU} / \mathrm{mL}$ and $100 \mu \mathrm{g} / \mathrm{mL})$. After 2 or 3 days, spheroidal aggregates were harvested and used for three-dimensional cultures.

Three-dimensional cultures of hepatocytic spheroids within the collagen gel matrix were performed using a type I collagen solution (Cellmatrix type I-A; Nitta Gelatin, Japan) as previously described. ${ }^{7}$ After being embedded within the gel, spheroidal aggregates were cultured in Williams' Medium E supplemented with $10 \mathrm{mmol} / \mathrm{L}$ nico- 
tinamide, $10 \%$ fetal bovine serum, $10^{-7} \mathrm{~mol} / \mathrm{L}$ insulin, 10 $\mathrm{ng} / \mathrm{mL}$ epidermal growth factor, and $10 \mathrm{ng} / \mathrm{mL}$ TNF- $\alpha$ (R\&D Systems, Minneapolis, MN).

To examine the reversibility of transdifferentiation, the ductular cells induced within the collagen gel were isolated by brief treatment with collagenase, transferred to multi-well plates coated with Matrigel (Becton-Dickinson), and cultured in serum-free Williams' Medium E supplemented with $10 \mathrm{mmol} / \mathrm{L}$ nicotinamide, $10 \mathrm{ng} / \mathrm{mL}$ epidermal growth factor, and $10^{-7} \mathrm{~mol} / \mathrm{L}$ insulin. Dex $(1 \mu \mathrm{mol} / \mathrm{L}$; Sigma-Aldrich) and $10 \mathrm{ng} / \mathrm{mL}$ of various cytokines (IL-6, $\mathrm{IL}-1 \alpha, \mathrm{IL}-1 \beta$, and oncostatin M; R\&D Systems, Inc.) were added to the medium. In some experiments, a specific inhibitor of c-Jun N-terminal kinase (SP600125; 20 $\mu \mathrm{mol} / \mathrm{L}$; Calbiochem, San Diego, CA) or mitogen-activated protein kinase (PD98059; $20 \mu \mathrm{mol} / \mathrm{L} ;$ Calbiochem) was added to the medium to examine the effect of the suppression of these signaling pathways.

DsRed2 fluorescence intensity, which parallels albumin expression, was monitored with a fluorescence plate reader (Cytofluor, PerSeptive Biosystems, Framingham, MA). The DsRed2 signal was excited at $530 \mathrm{~nm}$, and emission was detected at $620 \mathrm{~nm}$.

\section{Quantitative RT-PCR Analysis}

Total RNA from freshly isolated and cultured hepatocytes was extracted using TRIzol reagent (Life Technologies Japan, Tokyo, Japan). Quantitative RT-PCR was performed by the $\Delta \Delta$ Ct method using a QuantiTect SYBR Green RT-PCR Kit (Qiagen, Inc., Chatsworth, CA) and an Applied Biosystems StepOnePlus real-time PCR system (Life Technologies Japan). After reverse transcription at $50^{\circ} \mathrm{C}$ for 30 minutes and subsequent activation of HotStarTaq DNA polymerase at $95^{\circ} \mathrm{C}$ for 15 minutes, the cDNA was amplified with 40 cycles of a three-step PCR $\left(94^{\circ} \mathrm{C}\right.$ for 15 seconds, $60^{\circ} \mathrm{C}$ for 30 seconds, $72^{\circ} \mathrm{C}$ for 30 seconds). The specific primers were purchased from Qiagen (QuantiTect Primer Assays, Qiagen; albumin, QT00189679; TAT, QT00182308; glucose 6-phosphatase, QT00185948; phosphoenolpyruvate carboxykinase, QT01619975; bile salt export pump, QT00386008, cytokeratin [CK]19, QT00376887; sex determining region Y-box (Sox9), QT00427602; osteopontin, QT00199101; secretin receptor, QT00188426; cystic fibrosis transmembrane conductance regulator, QT01612289; glyceraldehyde 3-phosphate dehydrogenase, QT00199633). The sequences of primers for hepatocyte nuclear factor $4 \alpha$ (HNF-4 $\alpha$ ) were as follows: forward, 5'-GAAAATGTGCAGGTGTTGACCA-3'; reverse, 5'AGCTTGAGGCTCCGTAGTGTT-3'. The mRNA levels were normalized using glyceraldehyde 3-phosphate dehydrogenase $(\mathrm{GAPDH})$ as a housekeeping gene.

\section{Western Blot Analysis}

Cells cultured within the collagen gel and Matrigel were retrieved by a brief incubation with collagenase and the Cell Recovery Solution (Becton-Dickinson), respectively, and lysed in radioimmunoprecipitation assay buffer. Protein samples (30 $\mu \mathrm{g}$ of protein per lane) were subjected to SDS-PAGE using $10 \%$ polyacrylamide gels and were analyzed as previously described. ${ }^{7}$ The primary antibod- ies used were anti-albumin (Nordic Immunological Laboratories, Tilburg, Netherlands), anti-CK19 (a gift from Dr. Atsushi Miyajima, Institute of Molecular and Cellular Biosciences, the University of Tokyo), anti-TAT (EPR6121, Novus Biologicals), anti-Sox9 (AB5535, Millipore Corporation, Billerica, MA), anti-HNF-4 $\alpha$ (K9218; Perseus Proteomics, Tokyo, Japan), anti-HNF-1 (sc-8986; Santa Cruz Biotechnology, CA), anti-CCAAT/enhancer-binding protein (C/EBP) $\alpha$ (sc-61; Santa Cruz Biotechnology), anti-C/ $\operatorname{EBP} \beta$ (sc-150; Santa Cruz Biotechnology), anti-phosphop44/42 mitogen-activated protein kinase (ERK1/2) (Thr202/Tyr204) (No. 9101, Cell Signaling Technology, Beverly, MA), anti-p44/42 mitogen-activated protein kinase (ERK1/2) (No. 9102, Cell Signaling Technology), anti-phospho-stress-activated protein kinase/c-Jun N-terminal kinase (Thr183/Tyr185) (No. 9251, Cell Signaling Technology), anti-SAPK/JNK (No. 9258, Cell Signaling Technology), and anti- $\beta$-actin (Novus Biologicals).

\section{Histochemical and Morphological Examination}

Cultured cells and liver tissues were fixed in 10\% formaldehyde in $0.1 \mathrm{~mol} / \mathrm{L}$ phosphate-buffered saline $(\mathrm{pH}$ 7.4), embedded in paraffin, and sectioned. The sections were stained with H\&E. Periodic acid-Schiff stain (PAS), PAS with diastase digestion, and Sirius red staining were also performed in some experiments. Immunocytochemical or immunohistochemical staining was performed on deparaffinized sections treated with Target Retrieval Solution (DAKO Japan, Tokyo, Japan) using an anti-HNF-4 $\alpha$ antibody (K9218; Perseus Proteomics), anti-glutamine synthetase (ab73593, Abcam, Cambridge, UK), anti-CK19 (a gift from Dr. Atsushi Miyajima, Institute of Molecular and Cellular Biosciences, the University of Tokyo, Tokyo, Japan), and Histofine-Max-PO for rat tissues (Nichirei Biosciences, Tokyo, Japan). In double staining with anti-glutamine synthetase antibody and anti-CK19 antibody, Histogreen (Linaris Biologische Produkte, Dossenheim, Germany) and 3,3'-diaminobenzidine were used as peroxidase chromogens. Immunofluorescence staining for Thy 1 (CD90) (Novus Biologicals) was performed on smears of mature hepatocytes and fetal liver cells (E17.5) fixed by phosphate-buffered 4\% paraformaldehyde. Antibody binding was detected with fluorescein isothiocyanate-labeled anti-rabbit antibody (DAKO Japan). For the observation of the ultrastructures, cells were fixed with $2.5 \%$ glutaraldehyde and $1 \%$ osmium tetroxide and embedded in Epon resin. Ultrathin sections were stained with uranylate and lead, and observed under an electron microscope (JEOL, Tokyo, Japan).

\section{cDNA Microarray Analysis}

We compared the gene expression profiles of transdifferentiated and re-differentiating cells using the $4 \times 44 \mathrm{~K}$ Whole Rat Genome Oligo Microarray (Agilent Technologies Japan, Tokyo, Japan). Total RNA was extracted from freshly isolated hepatocytes, transdifferentiated hepatocytes cultured within the collagen gel for 14 days in the 

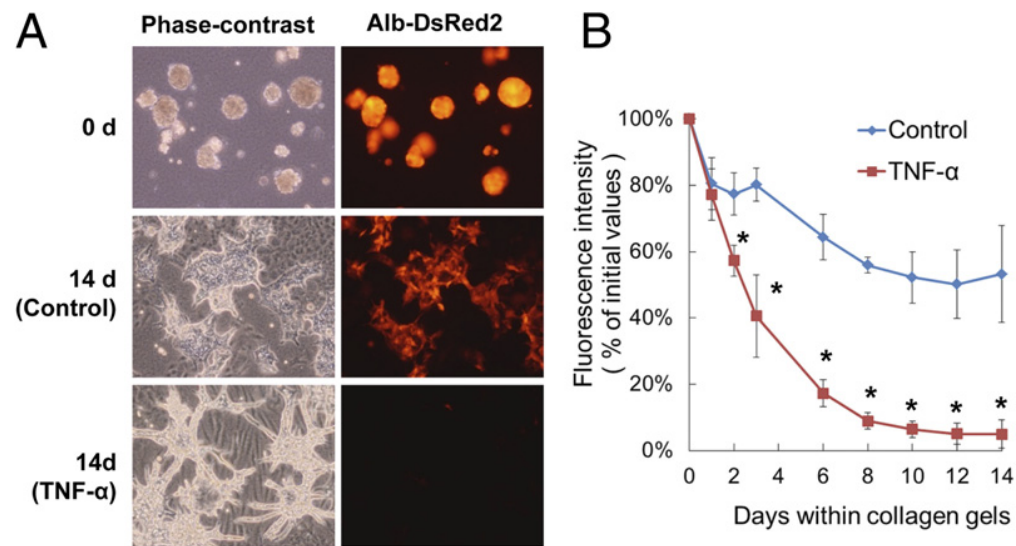

Figure 1. Transdifferentiation of Albumin (Alb)-DsRed2 transgenic rat hepatocytes within a collagen gel matrix. A: Phase-contrast microscopy and corresponding DsRed2 fluorescence of hepatocytic spheroids immediately after being embedded within a collagen gal matrix $[0$ days $(0 \mathrm{~d})]$ anc cultured for 14 days in the absence or presence of $10 \mathrm{ng} / \mathrm{mL}$ TNF- $\alpha$. B: The time course of DsRed 2 fluorescence intensity of the hepatocytic spheroids in collagen gel culture $(n=4)$. ${ }^{*} P<0.01$, compared with the control. C: Quantitative RTPCR analysis of the expression of hepatocyte-specific [albumin, HNF- $4 \alpha$, TAT, glucose 6-phosphatase (G6Pase), phosphoenolpyruvate carboxykinase (PEPCK), bile salt export pump (BSEP)] and bile duct/ductule-specific [CK19, Sox9, osteopontin (OPN)] genes in freshly isolated hepatocytes and in culture. Bars indicate the SD in a representative experiment performed in triplicate. Heps, hepatocytes.


presence of TNF- $\alpha$, cells cultured on Matrigel-coated surfaces for 7 days in the presence of IL-6 and Dex, and cells cultured on Matrigel-coated surfaces for 7 days in the presence of OSM and Dex. The gene expression profile of the transdifferentiated ductular cells was compared with that of the other samples, the genes that were upregulated more than fivefold or were down-regulated less than 0.2-fold were extracted, and the gene ontology (GO) category analysis and the hierarchical clustering were performed.

\section{Intrahepatic Transplantation of Transdifferentiated Hepatocytes}

To examine the reversibility of transdifferentiation in vivo, fully transdifferentiated ductular cells, which were cultured within the collagen gel for 14 days in the presence of TNF- $\alpha$, were transplanted into normal Wistar rat livers. Approximately $1 \times 10^{7}$ cells were re-suspended in $0.5 \mathrm{ml}$ of phosphate-buffered saline and injected via a mesenteric vein. After 7 or 10 days, the animals were sacrificed and the livers were perfused with phosphate-buffered $4 \%$ paraformaldehyde. Frozen sections were stained with Hoechst 33342 and observed by fluorescence microscopy. We performed two independent experiments.

\section{Results}

Phenotypic Transition of Adult Hepatocytes into Bile Ductular Cells within a Collagen Gel Matrix in the Presence of TNF- $\alpha$

The spheroidal aggregates of Albumin-DsRed2 hepatocytes emitted strong DsRed2 fluorescence that gradually decreased after being embedded within a collagen gel matrix, and the loss of fluorescent intensity was more rapid and profound when TNF- $\alpha$ was present in the medium (Figure 1, A and B). The loss of fluorescence was accompanied by a prominent branching morphogenesis (Figure 1A) and an increase in proliferating cell nuclear antigen (Nishikawa et al, unpublished data), but not by cell depletion due to cell death (see Supplemental Figure S2 at http://ajp.amjpathol.org). Quantitative RT-PCR analyses demonstrated that the expression of the hepatocytic differentiation marker genes (albumin, HNF-4 $\alpha$, TAT, glucose 6-phosphatase, phosphoenolpyruvate carboxykinase, and bile salt export pump) rapidly decreased during the period of spheroid culture and that they were virtually lost at the end of collagen gel culture (Figure 1C). In contrast, the expression of bile duct/ductular marker genes (CK19, Sox9, and osteopontin), which was completely absent in freshly isolated hepatocytes, increased during collagen gel culture (Figure 1C). The transdiffer- 

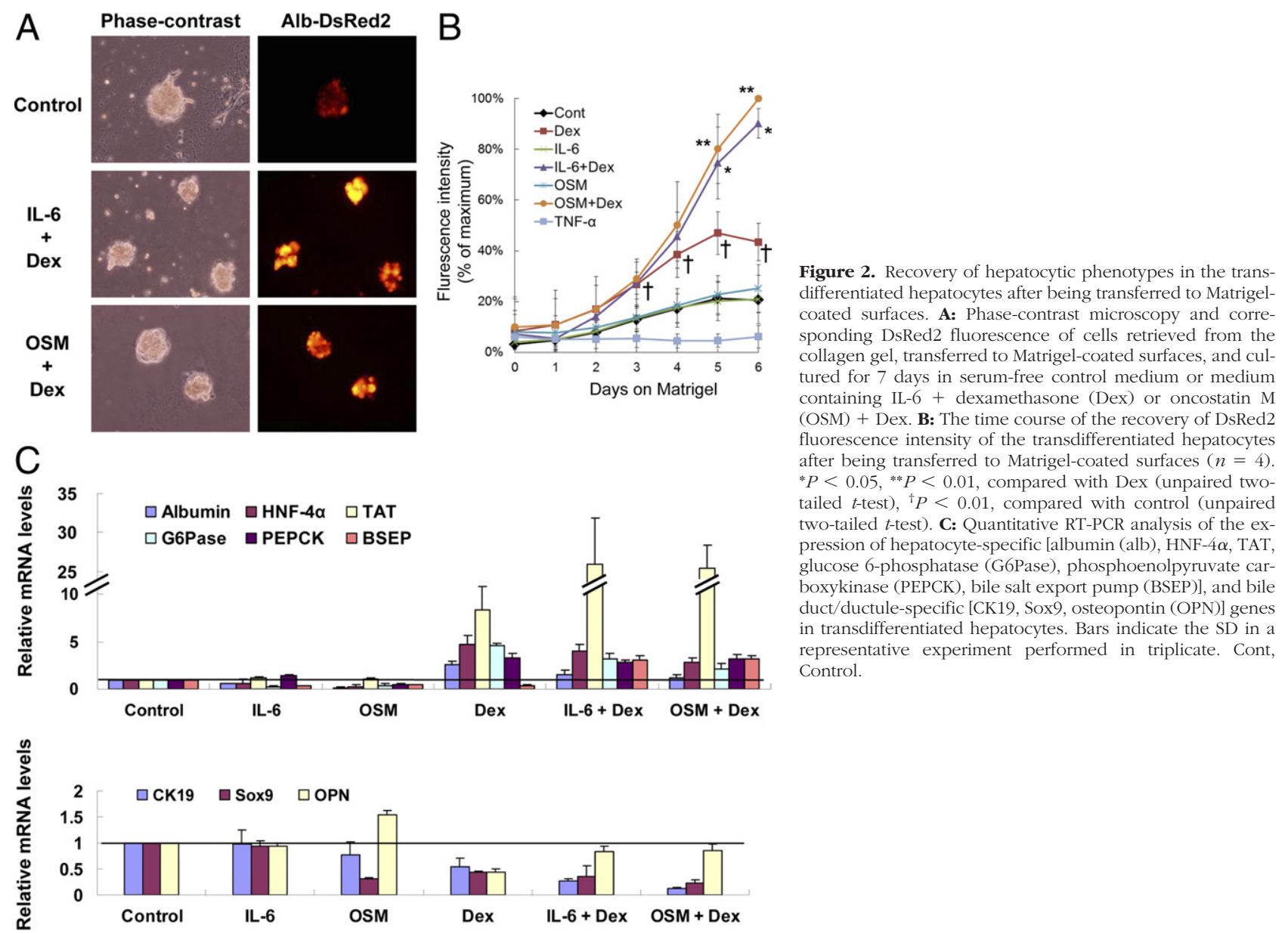

entiated hepatocytes expressed secretin receptor mRNA at high levels and cystic fibrosis transmembrane conductance regulator mRNA at low levels (see Supplemental Figure S3 at $h t t p: / / a j p . a m j p a t h o l . o r g)$, indicating that they could exhibit some of the bile duct or cholangiocyte phenotype, although their morphology was more similar to bile ductular cells. Flow cytometric and immunofluorescence analyses confirmed that cells expressing putative liver stem cell markers were virtually absent in the initial hepatocytic fraction (see Supplemental Figures S4 and S5 at http://ajp.amjpathol.org).

\section{Recovery of Hepatocytic Phenotypes in Transdifferentiated Ductular Cells after Transfer to Matrigel-Coated Surfaces and the Effects of Dex and Various Cytokines}

Transdifferentiated ductular cells in the presence of TNF- $\alpha$ were retrieved from the collagen gel matrix by collagenase treatment and were cultured on Matrigelcoated surfaces under serum-free conditions. The cells spontaneously formed spheroid aggregates and gradually recovered DsRed2 fluorescence, and this recovery was facilitated by Dex (Figure 2, A and B). Although the cytokines examined did not have significant effects on fluorescence recovery if applied alone, IL-6 and OSM remarkably enhanced the recovery synergistically with Dex (Figure 2, A and B).

The recovery of fluorescence on Matrigel was associated with the recovery of the expression of hepatocytic differentiation marker mRNA (Figure 2C). Dex increased the expression of albumin, HNF- $4 \alpha$, TAT, glucose 6-phosphatase, and phosphoenolpyruvate carboxykinase but not bile salt export pump (Figure 2C). Although IL-6 and OSM alone only slightly inhibited the expression of hepatocytic differentiation markers, they stimulated the expression of TAT and bile salt export pump mRNA when added together with Dex (Figure 2C). In contrast, the mRNA expression of bile ductular differentiation markers decreased in the presence of Dex, and this suppression was significantly augmented by IL-6 or OSM (Figure 2C). Western blot analysis demonstrated that Dex, especially when applied in combination with IL-6 or OSM, enhanced the protein expression of hepatocytic differentiation markers, while inhibited bile ductular differentiation markers (see Supplemental Figure S6 at http://ajp.amjpathol.org).

We next examined the protein expression of transcription factors that are known to be involved in hepatocytic differentiation. Although freshly isolated hepatocytes expressed HNF- $4 \alpha, \mathrm{HNF}-1 \alpha$, and $\mathrm{C} / \mathrm{EBP} \alpha$ at high levels, this expression dramatically declined after the initiation of collagen gel culture and was almost extinguished at the 


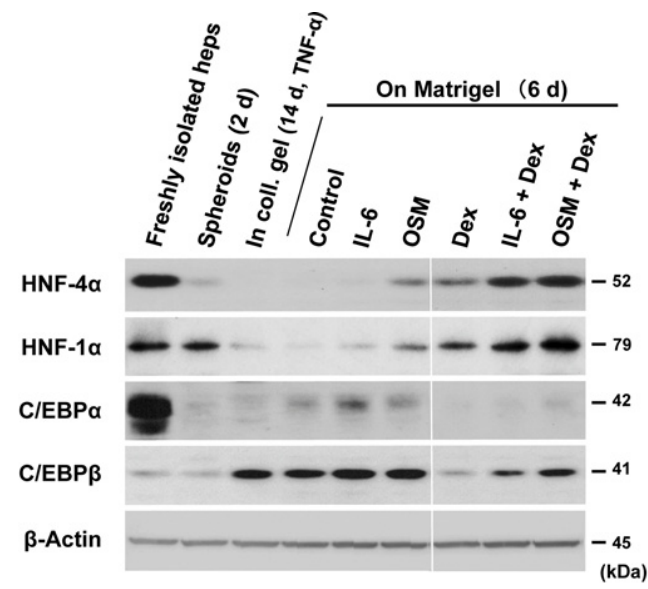

$\mathrm{B}$

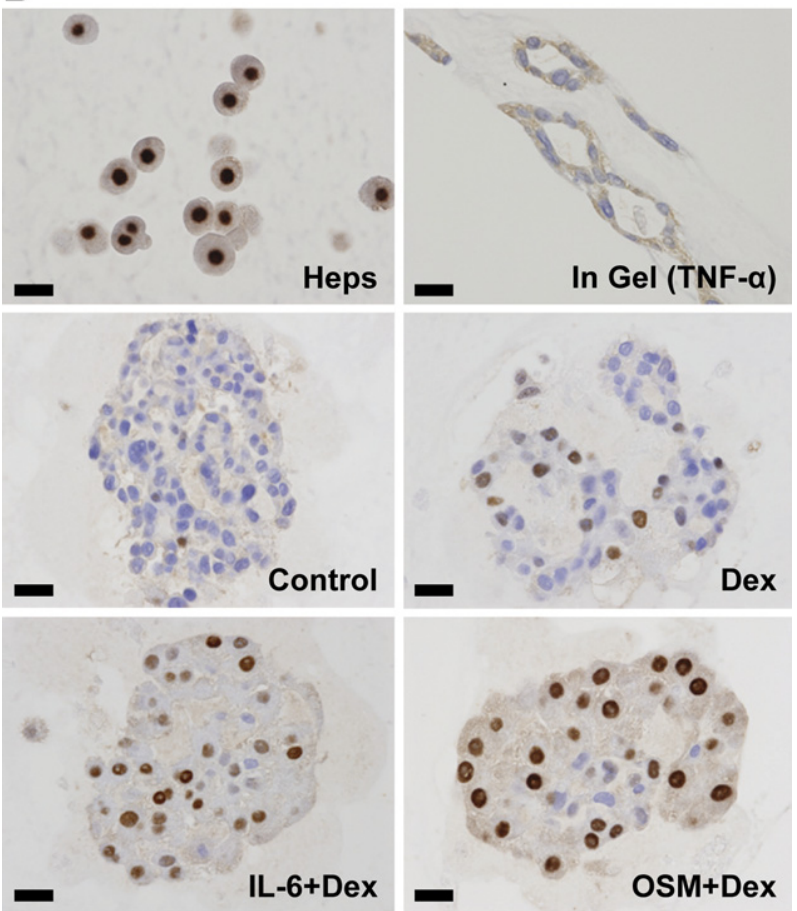

Figure 3. Recovery of the expression of hepatocyte-related transcription factors in the re-differentiating cells on Matrigel-coated surfaces. A: Western blot analysis of expression of HNF- $4 \alpha$, HNF- $1 \alpha$, CCAAT/enhancer-binding protein $\alpha$, and CCAAT/enhancer-binding protein $\beta$. Protein was extracted from freshly isolated hepatocytes, spheroids (cultured for 2 days on Primaria dishes), transdifferentiated hepatocytes (cultured within a collagen gel matrix in the presence of TNF- $\alpha$ for 14 days), and re-differentiating cells cultured on Matrigel-coated surfaces for 6 days in the absence (control) or presence of dexamethasone (Dex), IL-6, oncostatin M (OSM), IL-6 + Dex, or OSM + Dex, and analyzed. B: HNF- $4 \alpha$ immunocytochemistry. Freshly isolated hepatocytes (Heps), transdifferentiated hepatocytes cultured within a collagen matrix in the presence of TNF- $\alpha$ for 14 days [In Gel (TNF- $\alpha$ )], and re-differentiating hepatocytes cultured for 6 days on Matrigel in the control medium (control) or medium containing Dex, IL-6 + Dex, or OSM + Dex. C/EBP, anti-CCAAT/enhancer-binding protein. Scale bars: $20 \mu \mathrm{m}$.

end of the culture (Figure 3A). Interestingly, after being transferred onto Matrigel-coated surfaces, HNF- $4 \alpha$ and HNF-1 $\alpha$ expression was recovered, particularly when IL-6 or OSM was added together with Dex (Figure 3A; for $\mathrm{HNF}-4 \alpha$ ) (see Supplemental Figure S6 at http://ajp. amjpathol.org). Although $\mathrm{C} / \mathrm{EBP} \alpha$ expression slightly increased after the transfer, Dex inhibited this expression (Figure 3A). C/EBP $\beta$ expression, which was low in hepa- tocytes and increased during the culture period, was inhibited by Dex but slightly enhanced by IL-6 and OSM (Figure 3A).

Because these results suggested that $\mathrm{HNF}-4 \alpha$ protein expression was closely associated with the recovery of hepatocytic phenotypes, we performed immunocytochemistry to detect HNF- $4 \alpha$. Whereas freshly isolated hepatocytes strongly expressed HNF- $4 \alpha$ protein in their nuclei, ductular cells induced by culturing within the collagen gel matrix with TNF- $\alpha$ completely lost this expression (Figure 3B). After transfer to Matrigel-coated surfaces, only a few nuclei became positive for $\mathrm{HNF}-4 \alpha$ in control cells (Figure 3B). Although Dex alone slightly increased the number of HNF- $4 \alpha$ expressing cells, this effect was markedly enhanced by the presence of IL-6 or OSM (Figure 3B).

\section{Recovery of Hepatocytic Phenotypes by Dex Is Associated With the Inhibition of MEK and JNK Pathways}

To explore the possible mechanisms of the recovery of hepatocytic phenotypes, we examined the phosphorylation status of several signaling proteins. ERK phosphorylation increased in culture and was enhanced by IL-6, OSM, and TNF- $\alpha$ but was strongly inhibited by Dex (Figure 4A). JNK2/3 was transiently phosphorylated in freshly isolated hepatocytes which could be explained by cell stress during the isolation steps (Figure 4A). Although JNK2/3 was again phosphorylated in collagen gel culture with TNF- $\alpha$, its phosphorylation level was decreased after transfer to Matrigel-coated surfaces, especially in the presence of Dex (Figure 4A). JNK1 was only slightly phosphorylated in freshly isolated hepatocytes, but it was markedly phosphorylated in collagen gel culture with TNF- $\alpha$ (Figure 4A). Although the phosphorylation level of JNK1 was reduced after transfer, it was enhanced by IL-6, OSM, and TNF- $\alpha$ (Figure 4A). Dex efficiently repressed the effect of these cytokines, apparently through the inhibition of JNK protein expression (Figure 4A).

Because the inhibition of the MEK-ERK and JNK-CJun pathways may be associated with the recovery of hepatocytic phenotypes, we examined whether a MEK inhibitor (PD98059) or a JNK inhibitor (SP600125) affected this recovery. These inhibitors indeed augmented the recovery of DsRed2 fluorescence after transfer to Matrigel-coated surfaces, and the effect of the inhibitors was more prominent than that of Dex alone (Figure 4B). However, the effects of the inhibitors on HNF- $4 \alpha$ expression levels and cell morphology were comparable to those by Dex alone (Figure 4C). While PD98059 slightly enhanced the recovery of albumin expression, these inhibitors did not significantly affect the protein expression levels of hepatocytic and bile ductular differentiation markers (see Supplemental Figure S6 at http://ajp.amjpathol.org). 
A
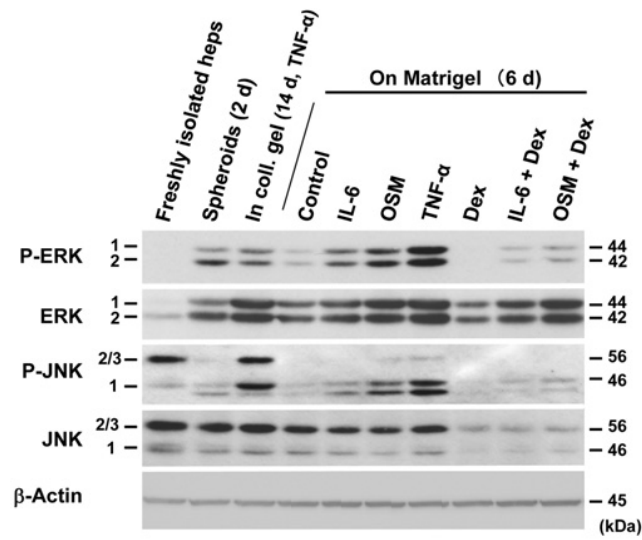

B

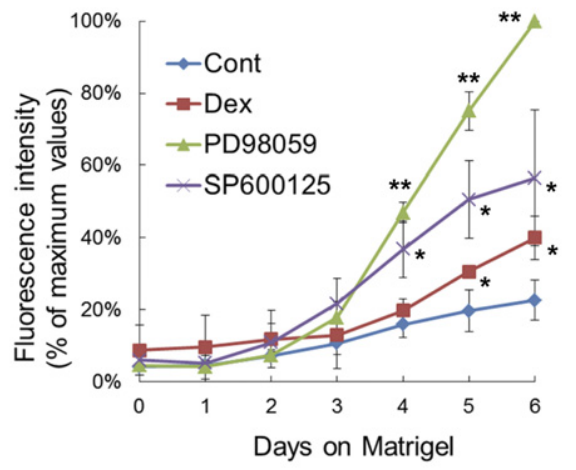

C


Figure 4. Dexamethasone (Dex)-induced suppression of the ERK and JNK phosphorylation in the re-differentiating cells on Matrigel-coated surfaces. A: Western blot analysis of total and phosphorylated ERK and JNK. Protein was extracted from freshly isolated hepatocytes, spheroids (cultured for 2 days on Primaria dishes), transdifferentiated hepatocytes (cultured within a collagen gel matrix in the presence of TNF- $\alpha$ for 14 days), and re-differentiating cells [cultured on Matrigel-coated surfaces for 6 days in the absence (control) or presence of Dex, IL-6, oncostatin M (OSM), TNF- $\alpha$, IL- 6 + Dex, or OSM + Dex] and analyzed. B: The time course of DsRed 2 fluorescence intensity of transdifferentiated hepatocytes on Matrigel-coated surfaces $(n=4)$ comparing the control, Dex, PD98059 (MEK inhibitor), and SP600125 (JNK inhibitor). ${ }^{*} P<0.05,{ }^{* *} P<0.01$, compared with control (unpaired two-tailed $t$-test). C: HNF- $4 \alpha$ immunocytochemistry of re-differentiating hepatocytes cultured on Matrigel-coated surfaces for 6 days in the presence of Dex, PD98059, or SP600125. Cont, Control; ERK, extracellular signal-regulated kinase; JNK, c-Jun $\mathrm{NH}_{2}$-terminal kinase; p-ERK, phosphorylated extracellular signal-regulated kinase; p-JNK, phosphorylated c-Jun $\mathrm{NH}_{2}$-terminal kinase. Scale bars: $20 \mu \mathrm{m}$.

\section{IL-6 + Dex or OSM + Dex Restores Mature Hepatocytic Morphology after Ductular Transdifferentiation of Hepatocytes}

Mature hepatocytes have one or two large nuclei with a prominent nucleolus and abundant eosinophilic cytoplasm, whereas interlobular bile ducts and bile ductules are composed of small cuboidal epithelial cells (Figure
$5 A)$. After being cultured within the collagen gel matrix with TNF- $\alpha$ for 14 days, hepatocytes transformed into small cuboidal cells forming small tubular structures, morphologically similar to bile ductules (Figure 5B). Although collagenase-digested and dispersed ductular cells formed aggregates on Matrigel-coated surfaces and recovered some hepatocytic markers, they were small, polygonal, and distinct from the original mature hepatocytes (Figure 5C). The addition of Dex, IL-6, or OSM alone did not affect the morphology (Figure 5, D and E). However, when IL-6 or OSM was added together with Dex, the majority of the cells was enlarged and exhibited mature hepatocytic features, including large nuclei, prominent nucleoli, and abundant eosinophilic cytoplasm (Figure 5, F and G). Furthermore, some of these cells accumulated glycogen, which was demonstrated by PAS and PAS with diastase digestion staining (Figure $5, \mathrm{H}$ and $\mathrm{I})$.
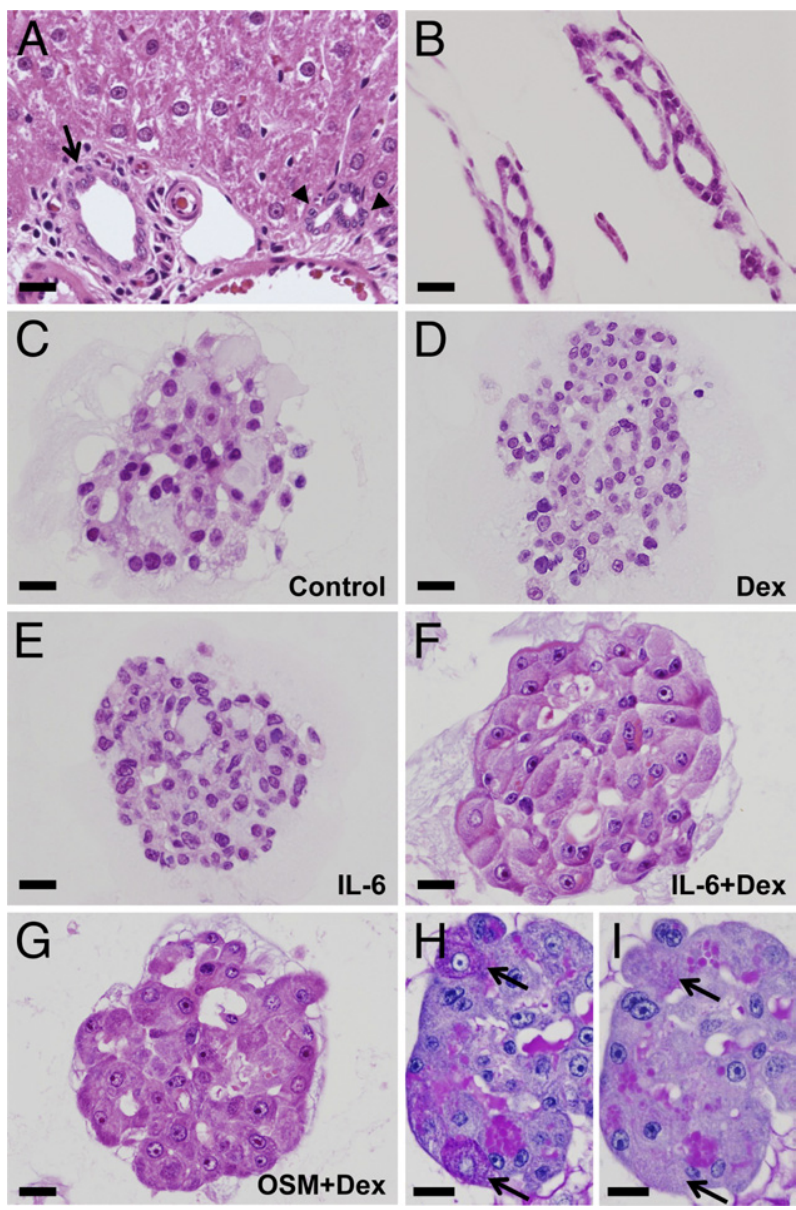

Figure 5. Mature hepatocytic morphology in re-differentiating hepatocytes induced by IL- 6 and oncostatin M (OSM) in the presence of dexamethasone (Dex). H\&E staining (A-G), periodic acid-Schiff stain $(\mathbf{H})$, periodic acid-Schiff stain with diastase digestion staining (I). A: Normal Albumin-DsRed2 rat liver tissue. An interlobular bile duct (arrow) and two bile ductules (arrowheads) are seen. B: Transdifferentiated hepatocytes induced by culturing for 14 days within a collagen gel matrix in the presence of TNF- $\alpha$. $(\mathbf{C}-\mathbf{H})$ Re-differentiating hepatocytes cultured for 6 days on Matrigel-coated surfaces. C: Control, (D) Dex, (E) IL-6, (F) IL-6 + Dex, (G) OSM + Dex, (H, I) IL-6 + Dex (arrows indicate two identical cells in adjacent sections without and with diastase treatment). Scale bars: $20 \mu \mathrm{m}$. 

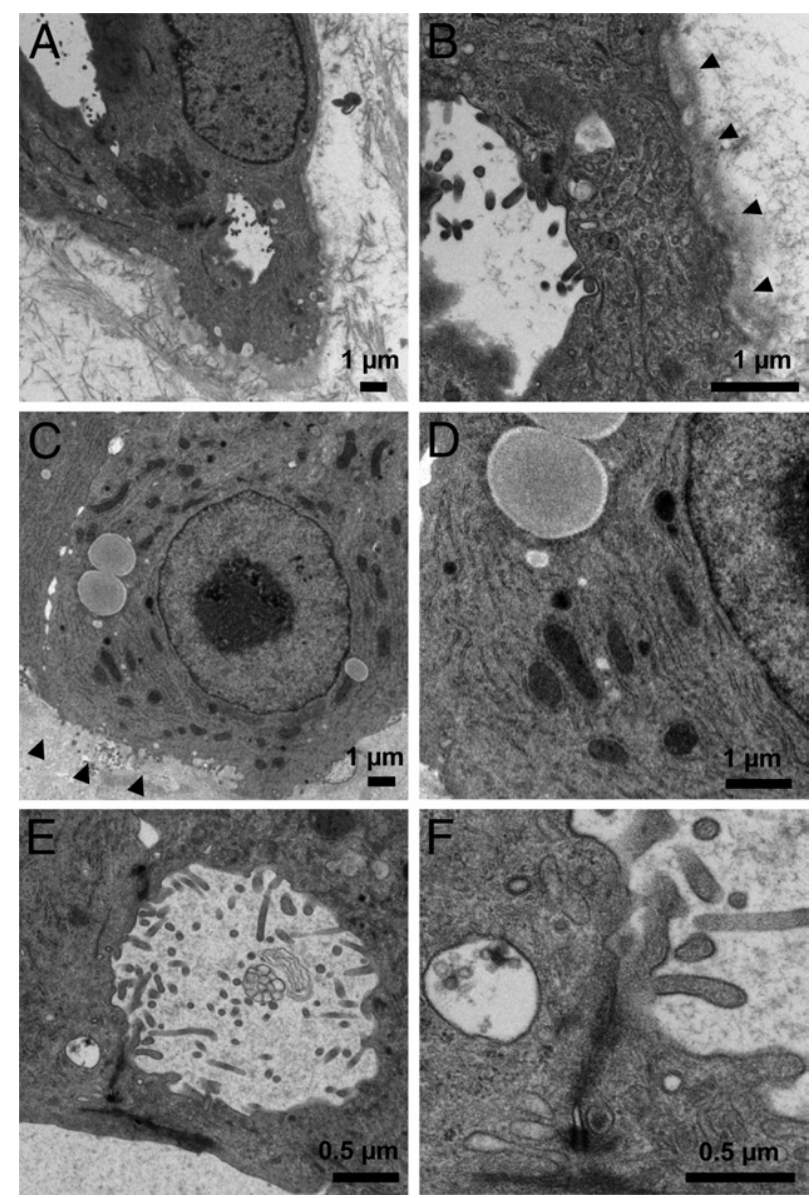

Figure 6. Appearance of mature hepatocytic ultrastructures in re-differentiating hepatocytes treated with IL-6 and dexamethasone (Dex). Uranylate and lead staining. A, B: Transdifferentiated hepatocytes induced by culturing for 14 days within a collagen gel matrix in the presence of TNF- $\alpha$. The arrowheads in $\mathbf{B}$ indicate basement membranes around the ductular structure. C-F: Re-differentiating hepatocytes cultured for 6 days on Matrigel-coated surfaces in the presence of IL-6 + Dex. The arrowheads in $\mathbf{C}$ indicate the surface of the cell lacking basement membranes.

The ultrastructural analysis of the branching ductular structures formed within the collagen gel matrix revealed that they were surrounded by basement membranes and possessed sparse microvilli on the luminal surfaces (Figure $6, A$ and $B$ ). In contrast, the redifferentiating cells on the Matrigel had microvilli on their surfaces, lacked basement membranes (Figure 6C), and had an extensive cytoplasm that contained many mitochondria, rough endoplasmic reticula, and a few lipid droplets (Figure 6D). Furthermore, they formed bile canaliculus-like lumens with well-developed cell adhesion complexes (Figure 6, E and F).

\section{The mRNA Expression Profile of}

Redifferentiating Hepatocytes Treated with IL-6 + Dex and OSM + Dex on Matrigel Is Similar to that of Freshly Isolated Hepatocytes

To analyze the level of redifferentiation into hepatocytes after transfer to Matrigel, we analyzed the mRNA extracted from freshly isolated hepatocytes, transdifferenti- ated hepatocytes within the collagen gel, and redifferentiating hepatocytes on Matrigel in the presence of IL-6 + Dex or OSM + Dex by a cDNA microarray. Gene ontology analysis revealed that the transdifferentiation process was associated with changes in the gene products of various gene ontology terms and, interestingly, changes in the opposite directions were found in the re-differentiation process (see Supplemental Table S1 at http://ajp.amjpathol.org). Genes that were upregulated more than fivefold and were down-regulated less than 0.2 -fold were selected and analyzed. The amounts of differentially expressed mRNAs were 4849, 1762, and 1602 for freshly isolated hepatocytes, re-differentiating hepatocytes treated with IL-6 + Dex, and those treated with OSM + Dex, respectively (Figure 7). A Venn diagram analysis identified 799 differentially expressed genes common to the three samples (Figure 7), and a hierarchical clustering analysis was performed for these genes. The heat map showed that the pattern in the transdifferentiated hepatocytes was quite different from that of freshly isolated hepatocytes, whereas the re-differentiating hepatocytes treated either by IL6 + Dex or OSM + Dex generated patterns similar to that of freshly isolated hepatocytes (Figure 7). The Pearson's correlation coefficients of IL-6 + Dex- versus OSM + Dex-treated regenerating hepatocytes, IL-6 + Dex-treated regenerating hepatocytes versus freshly isolated hepatocytes, and IL-6 + Dex-treated regenerating hepatocytes versus transdifferentiated hepatocytes were $0.98,0.75$, and 0.24, respectively (Figure 7 ).

\section{Evidence for Reversion of Transdifferentiated Phenotype of Hepatocytes in Vivo}

We previously demonstrated that altered phenotypes in 5 day-cultured hepatocytic spheroids were more efficiently reversed after intrahepatic transplantation as compared with intrasplenic transplantation. ${ }^{16}$ To examine whether fully transdifferentiated hepatocytes could reverse their
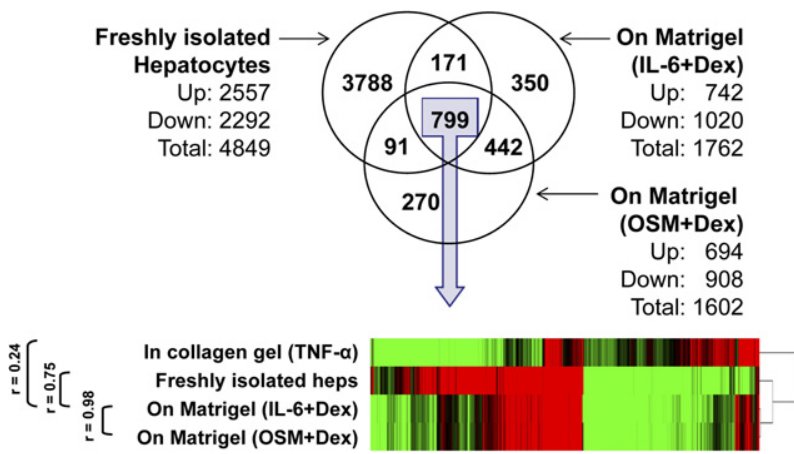

Figure 7. Recovery of the mRNA expression profile of mature hepatocytes by culturing on Matrigel-coated surfaces in the presence of IL- $6+$ dexamethasone (Dex) or oncostatin M (OSM) + Dex. A cDNA microarray analysis of mRNA extracted from freshly isolated hepatocytes, transdifferentiated hepatocytes induced by culturing for 14 days within a collagen gel matrix in the presence of TNF- $\alpha$ and re-differentiating hepatocytes for 6 days on Matrigel in the presence of IL-6 + Dex or OSM + Dex. Differentially expressed genes common to the three samples (799 genes) were subject to a hierarchical clustering analysis. Pearson's correlation coefficients are indicated at the left of the heat map. 

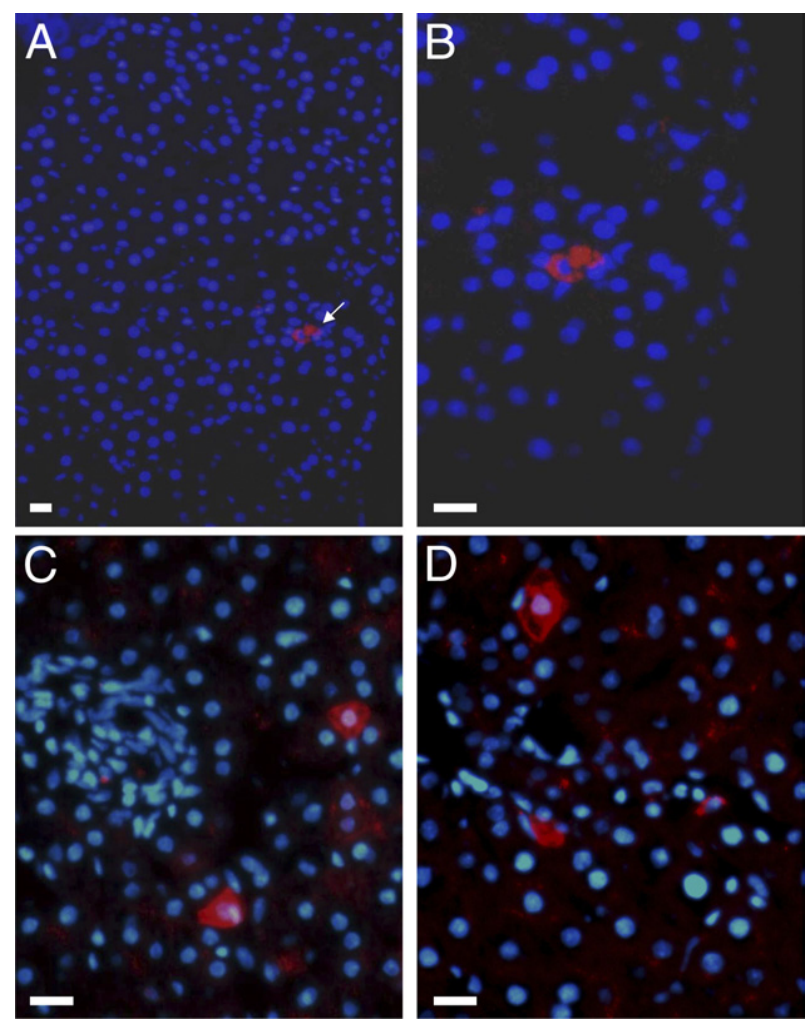

Figure 8. Evidence for reversibility of ductular transdifferentiation of hepatocytes in vivo. Fluorescence microscopy of rat livers 7 days after transplantation of transdifferentiated Albumin-DsRed2 hepatocytes, which were cultured within the collagen gel for 14 days with TNF- $\alpha$. Merged images of DsRed2 fluorescence and nuclear staining with Hoechst 33342 (A, B) or DAP $(\mathbf{C}, \mathbf{D})$ from two independent experiments. The arrow in $\mathbf{A}$ indicates DsRed2 fluorescent cells, which are enlarged in $\mathbf{B}$. Scale bars: $20 \mu \mathrm{m}$.

original phenotype in the normal liver environment, we transplanted transdifferentiated Albumin-DsRed2 hepatocytes, which were cultured within the collagen gel matrix for 14 days in the presence of TNF- $\alpha$, into intact livers of Wistar rats via the portal vein. After 7 or 10 days, scattered large hepatocytes emitting DsRed2 fluorescence were reproducibly observed in the liver parenchyma (Figure 8), suggesting the reversible nature of ductular transdifferentiation in a normal liver microenvironment.

\section{Discussion}

In this study, we demonstrated that transdifferentiated hepatocytes in vitro could recover the original hepatocytic phenotypes if they were placed in a more physiological condition. The transdifferentiated cells retrieved from the collagen gel matrix spontaneously formed spheroidal aggregates on Matrigel-coated surfaces. In our preliminary experiments, cells plated with a serum-containing medium spread across the surface without forming spheroids, and there was no recovery of DsRed2 fluorescence. Cultures of hepatocytes in spheroidal aggregates have been shown to be beneficial in the maintenance of differentiated phenotypes of hepatocytes. ${ }^{17,18}$ Thus, spheroid formation appeared to be important for the recovery of hepatocytic phenotypes, probably via increasing cell-cell contacts in self-organized three-dimensional structures. Furthermore, the interaction of cells with the components of the Matrigel might play important roles in the recovery partly through the modulation of expression of transcription factors, including HNF- $4 \alpha,{ }^{19}$ and the activation of integrin-linked kinase, which is involved in transmitting integrin signaling to the interior of the cell. ${ }^{20,21}$

The recovery of albumin expression in transdifferentiated hepatocytes was enhanced by Dex, which has been shown to increase albumin and TAT expression in spheroidal aggregate cultures of newborn rat hepatocytes ${ }^{18}$ and decrease CK19 mRNA expression in monolayer-cultured rat hepatocytes. ${ }^{7}$ Although the mechanism of the effect of Dex on hepatocytic phenotypes has not been elucidated, this study suggests that the inhibition of the MEK and JNK signaling pathways may be important. In a previous study, we also found that PD98059 inhibited aberrant CK19 expression in hepatocytes cultured within the collagen gel matrix. ${ }^{7}$ Dex inhibits the phosphorylation of JNK via a direct interaction with the glucocorticoid receptor in rat hepatocytes. ${ }^{22}$ However, interestingly, Dex decreased JNK at the protein level in our experiments, suggesting that this may be a long-term effect of Dex in the regulation of JNK activity.

Although the phenotypic reversion toward hepatocytes induced by Dex was limited, it was markedly enhanced by IL-6 and OSM, which belong to the IL-6 family of cytokines. Our results illustrate the differential effects of inflammatory cytokines on the regulation of differentiation between hepatocytes and bile ductules: IL-6 and OSM facilitate or maintain hepatocytic differentiation, whereas $\mathrm{TNF}-\alpha$ induces the bile ductular transdifferentiation of hepatocytes. The in vitro maturation of fetal mouse hepatoblasts is accomplished by the addition of Dex with OSM, through the activation of gp $130,{ }^{14}$ which is crucial for the maturation of hepatocytes. Furthermore, fetal mouse hepatoblasts are able to fully mature with the expression of tryptophan oxygenase in high-cell-density culture with Matrigel in the presence of Dex and OSM. ${ }^{23}$ Interestingly, in contrast to the reversion of transdifferentiated hepatocytes, IL-6 is ineffective at inducing the differentiation of fetal hepatocytes toward mature hepatocytes in vitro. Although OSM can directly bind to gp130 on the target cells and then form a complex with leukemia inhibitory factor receptor, IL-6 needs to be bound to the IL-6 receptor, either the membrane-bound or soluble form, to associate with gp $130 .{ }^{24}$ Although IL-6 itself does not affect the proliferation of fetal rat hepatocytes, a chimera of $\mathrm{IL}-6$ and its soluble receptor stimulates their proliferation and maturation. ${ }^{25} \mathrm{IL}-6$ receptors may not express or function in fetal hepatocytes. Our results highlight both the similarities and differences between the maturation of fetal hepatocytes and the recovery of transdifferentiated hepatocytes.

One of the most impressive molecular events associated with the synergistic effects of Dex and IL-6 or OSM was the recovery of $\mathrm{HNF}-4 \alpha$ at the protein level. Although Dex alone enhanced HNF- $4 \alpha$ mRNA levels, there was not a significant associated increase in $\mathrm{HNF}-4 \alpha$ protein levels, suggesting the involvement of post-transcriptional 
control mechanisms. HNF- $4 \alpha$ protein levels can be modulated without a change in HNF- $4 \alpha$ mRNA expression in HepG2 cells. ${ }^{26,27}$ HNF- $4 \alpha$ is known to be one of the most important transcription factors that drive the differentiation of the hepatic lineage. ${ }^{28} \mathrm{HNF}-4 \alpha$ binding sites have been identified in many hepatocyte-specific genes that are involved in the metabolism of glucose, lipids, amino acids, and xenobiotics. ${ }^{29,30} \mathrm{~A}$ recent analysis using a new integrated approach identified approximately 240 direct human target genes, including both known and unexpected targets. ${ }^{31}$ Interestingly, the recovery of hepatocytic phenotypes induced by IL-6 + Dex or OSM + Dex was also associated with a marked increase in the HNF-1 $\alpha$ protein, another liver-specific transcription factor whose gene expression is known to be directly controlled by HNF- $4 \alpha{ }^{29}$ The correlated expression of these two liver-specific transcription factors has been observed in the well-differentiated status of primary rat hepatocytes cultured on Matrigel. ${ }^{32}$

In our three-dimensional culture model, hepatocytic spheroids transformed into ductular structures composed of small cuboidal cells, which were morphologically similar to bile ductules. However, after re-differentiation on Matrigel in the presence of IL-6 + Dex or OSM + Dex, they recovered the original morphology of mature hepatocytes with accumulated glycogen in their cytoplasm. The phenotypic recovery was further confirmed by cDNA microarray analysis, which demonstrated a significant reversal of the altered gene expression profiles that occurred after transdifferentiation. The mechanisms regulating the size of the hepatocytes are largely unknown. Although the phosphatidylinositol-3 kinase/Akt pathway has been implicated in the regulation of cell size $^{33}$ and has been shown to be responsible for hepatocyte hypertrophy during liver regeneration. ${ }^{34}$ Akt phosphorylation increased during transdifferentiation (Nishikawa et al, unpublished data). As suggested by experiments using conditional HNF- $4 \alpha$ knockout mice, in which small hepatocytes with decreased glycogen accumulation were found in the fetal liver (E18.5) with abnormally formed sinusoidal structures, ${ }^{35}$ the coordinated actions of molecular events induced by this transcription factor may be indispensable for the development, maintenance, and recovery of normal hepatocytic phenotype.

Our results suggest that hepatocytic and bile ductular phenotypes may be mutually reversible. Although the present study mainly focused on the in vitro experiments, we demonstrated that fully transdifferentiated AlbuminDsRed2 hepatocytes could recover their fluorescence and hepatocytic morphology when they settled in the normal liver environment. We also suggested that the centrilobular ductular reaction induced by chronic $\mathrm{CCl}_{4}$ injury might be reversible. Although the origin of ductular cells in the ductular reaction is still debated and a recent work did not find evidence for formation of ductular cells from hepatocytes, ${ }^{36}$ we have obtained clear evidence for the involvement of ductular transdifferentiation of mature hepatocytes in the ductular reaction, using several hepatocyte lineage tracing systems in mice (Nagahama et al, unpublished data). Our hypothesis of phenotypic plasticity between hepatocytes and bile ductular cells in the adult liver may have relevance to the disease process, as well as the recovery, in chronic liver diseases.

\section{Acknowledgments}

We thank Eiji Kobayashi for generously providing the Albumin-DsRed2 transgenic rats and Yasuharu Ueno for helpful suggestions regarding the genotyping of the transgenic rats. We appreciate the excellent technical assistance of Akiko Yagisawa, Reiko Ito, Makiko Kawamata, and Yoko Okada.

\section{References}

1. Desmet V, Roskams T, Van Eyken P: Ductular reaction in the liver. Pathol Res Pract 1995, 191:513-524

2. Alvaro D, Mancino MG, Glaser S, Gaudio E, Marzioni M, Francis H, Alpini G: Proliferating cholangiocytes: a neuroendocrine compartment in the diseased liver. Gastroenterology 2007, 132:415-431

3. Fausto N, Campbell JS: The role of hepatocytes and oval cells in liver regeneration and repopulation. Mech Dev 2003, 120:117-130

4. Duncan AW, Dorrell C, Grompe M: Stem cells and liver regeneration. Gastroenterology 2009, 137:466-481

5. Pai RK, Hart JA: Aberrant expression of cytokeratin 7 in perivenular hepatocytes correlates with a cholestatic chemistry profile in patients with heart failure. Mod Pathol 2010, 23:1650-1656

6. Desmet VJ: Ductal plates in hepatic ductular reactions. Hypothesis and implications. II. Ontogenic liver growth in childhood. Virchows Arch 2011, 458:261-270

7. Nishikawa Y, Doi Y, Watanabe H, Tokairin T, Omori Y, Su M, Yoshioka $\mathrm{T}$, Enomoto K: Transdifferentiation of mature rat hepatocytes into bile duct-like cells in vitro. Am J Pathol 2005, 166:1077-1088

8. Nishikawa $Y$, Tokusashi $Y$, Kadohama T, Nishimori H, Ogawa K: Hepatocytic cells form bile duct-like structures within a three-dimensional collagen gel matrix. Exp Cell Res 1996, 223:357-371

9. Block GD, Locker J, Bowen WC, Petersen BE, Katyal S, Strom SC Riley T, Howard TA, Michalopoulos GK: Population expansion, clonal growth, and specific differentiation patterns in primary cultures of hepatocytes induced by HGF/SF. EGF and TGF alpha in a chemically defined (HGM) medium. J Cell Biol 1996, 132:1133-1149

10. Michalopoulos GK, Barua L, Bowen WC: Transdifferentiation of rat hepatocytes into biliary cells after bile duct ligation and toxic biliary injury. Hepatology 2005, 41:535-544

11. Limaye PB, Bowen WC, Orr AV, Luo J, Tseng GC, Michalopoulos GK: Mechanisms of hepatocyte growth factor-mediated and epidermal growth factor-mediated signaling in transdifferentiation of rat hepatocytes to biliary epithelium. Hepatology 2008, 47:1702-1713

12. Schuetz EG, Li D, Omiecinski CJ, Muller-Eberhard U, Kleinman HK, Elswick B, Guzelian PS: Regulation of gene expression in adult rat hepatocytes cultured on a basement membrane matrix. J Cell Physiol 1988, 134:309-323

13. Kamiya A, Kinoshita T, Ito $Y$, Matsui T, Morikawa $Y$, Senba E, Nakashima K, Taga T, Yoshida K, Kishimoto T, Miyajima A: Fetal liver development requires a paracrine action of oncostatin $\mathrm{M}$ through the gp130 signal transducer. EMBO J 1999, 18:2127-2136

14. Kinoshita T, Sekiguchi T, Xu MJ, Ito Y, Kamiya A, Tsuji K, Nakahata T, Miyajima A: Hepatic differentiation induced by oncostatin $M$ attenuates fetal liver hematopoiesis. Proc Natl Acad Sci USA 1999, 96: 7265-7270

15. Sato $Y$, Igarashi $Y$, Hakamata $Y$, Murakami T, Kaneko T, Takahashi M, Seo N, Kobayashi E: Establishment of Albumin-DsRed2 transgenic rat for liver regeneration research. Biochem Biophys Res Commun 2003, 311:478-481

16. Nishikawa Y, Ohta T, Ogawa K, Nagase S: Reversion of altered phenotype in primary cultured rat hepatocytes after intrahepatic and intrasplenic transplantation. Lab Invest 1994, 70:925-932

17. Koide N, Sakaguchi K, Koide Y, Asano K, Kawaguchi M, Matsushima $\mathrm{H}$, Takenami T, Shinji T, Mori M, Tsuji T: Formation of multicellular spheroids composed of adult rat hepatocytes in dishes with positively 
charged surfaces and under other nonadherent environments. Exp Cell Res 1990, 186:227-235

18. Landry J, Bernier D, Ouellet C, Goyette R, Marceau N: Spheroidal aggregate culture of rat liver cells: histotypic reorganization, biomatrix deposition, and maintenance of functional activities. J Cell Biol 1985, 101:914-923

19. Oda H, Nozawa K, Hitomi $Y$, Kakinuma A: Laminin-rich extracellular matrix maintains high level of hepatocyte nuclear factor 4 in rat hepatocyte culture. Biochem Biophys Res Commun 1995, 212:800805

20. Gkretsi V, Bowen WC, Yang Y, Wu C, Michalopoulos GK: Integrinlinked kinase is involved in matrix-induced hepatocyte differentiation. Biochem Biophys Res Commun 2007, 353:638-643

21. Gkretsi V, Mars WM, Bowen WC, Barua L, Yang Y, Guo L, St-Arnaud R, Dedhar S, Wu C, Michalopoulos GK: Loss of integrin linked kinase from mouse hepatocytes in vitro and in vivo results in apoptosis and hepatitis. Hepatology 2007, 45:1025-1034

22. Ventura JJ, Roncero C, Fabregat I, Benito M: Glucocorticoid receptor down-regulates c-Jun amino terminal kinases induced by tumor necrosis factor alpha in fetal rat hepatocyte primary cultures. Hepatology 1999, 29:849-857

23. Kamiya A, Kojima N, Kinoshita T, Sakai Y, Miyaijma A: Maturation of fetal hepatocytes in vitro by extracellular matrices and oncostatin M: Induction of tryptophan oxygenase. Hepatology 2002, 35:1351-1359

24. Rose-John S, Scheller J, Elson G, Jones SA: Interleukin-6 biology is coordinated by membrane-bound and soluble receptors: role in inflammation and cancer. J Leukoc Biol 2006, 80:227-236

25. Zvibel I, Brill S, Kariv R, Traister A, Golan T, Chebath J, Halpern Z, Revel M, Oren R: Chimeric molecule IL-6/soluble IL-6 receptor is a potent mitogen for fetal hepatocytes. J Cell Physiol 2004, 200:245252

26. Wang B, Cai SR, Gao C, Sladek FM, Ponder KP: Lipopolysaccharide results in a marked decrease in hepatocyte nuclear factor 4 alpha in rat liver. Hepatology 2001, 34:979-989
27. Ying $C$, Li $Y$, Leung $C H$, Robek MD, Cheng YC: Unique antiviral mechanism discovered in anti-hepatitis $B$ virus research with a natural product analogue. Proc Natl Acad Sci USA 2007, 104: $8526-8531$

28. Nagaki M, Moriwaki H: Transcription factor HNF and hepatocyte differentiation. Hepatol Res 2008, 38:961-969

29. Watt AJ, Garrison WD, Duncan SA: HNF4: a central regulator of hepatocyte differentiation and function. Hepatology 2003, 37:12491253

30. Odom DT, Zizlsperger N, Gordon DB, Bell GW, Rinaldi NJ, Murray HL, Volkert TL, Schreiber J, Rolfe PA, Gifford DK, Fraenkel E, Bell Gl, Young RA: Control of pancreas and liver gene expression by HNF transcription factors. Science 2004, 303:1378-1381

31. Bolotin E, Liao H, Ta TC, Yang C, Hwang-Verslues W, Evans JR, Jiang T, Sladek FM: Integrated approach for the identification of human hepatocyte nuclear factor 4 alpha target genes using protein binding microarrays. Hepatology 2010, 51:642-653

32. Nagaki M, Shidoji $Y$, Yamada $Y$, Sugiyama A, Tanaka M, Akaike T, Ohnishi H, Moriwaki H, Muto Y: Regulation of hepatic genes and liver transcription factors in rat hepatocytes by extracellular matrix. Biochem Biophys Res Commun 1995, 210:38-43

33. Kozma SC, Thomas G: Regulation of cell size in growth, development and human disease: pl3K. PKB and S6K. Bioessays 2002, 24:65-71

34. Haga S, Ogawa W, Inoue H, Terui K, Ogino T, Igarashi R, Takeda K, Akira S, Enosawa S, Furukawa H, Todo S, Ozaki M: Compensatory recovery of liver mass by Akt-mediated hepatocellular hypertrophy in liver-specific STAT3-deficient mice. J Hepatol 2005, 43:799-807

35. Parviz F, Matullo C, Garrison WD, Savatski L, Adamson JW, Ning G, Kaestner KH, Rossi JM, Zaret KS, Duncan SA: Hepatocyte nuclear factor 4alpha controls the development of a hepatic epithelium and liver morphogenesis. Nat Genet 2003, 34:292-296

36. Malato Y, Naqvi S, Schurmann N, Ng R, Wang B, Zape J, Kay MA, Grimm D, Willenbring H: Fate tracing of mature hepatocytes in mouse liver homeostasis and regeneration. J Clin Invest 2011, 121:48504860 